\title{
The angle of visual roll motion determines displacement of subjective visual vertical
}

\author{
K. H. MAURITZ, J. DICHGANS, and A. HUFSCHMIDT \\ Abteilund für Klinische Neurologie und Neurophysiologie der Universität \\ D-7800 Freiburg i. Br., Hansastrasse 9, West Germany
}

\begin{abstract}
The effect of full-field sinusoidal visual roll motion stimuli of various frequencies and peak velocities upon the orientation of subjective visual vertical (SV) was studied. The angle of the optokinetically induced displacement of SV was found to be a linear function of the logarithm of the stimulus oscillation angle. Interindividual slopes of this function varied between 2 and 9 . The logarithmic function is independent of stimulus frequency within the range of $.02 \mathrm{~Hz}$ to $.5 \mathrm{~Hz}$ and of peak stimulus velocity from $7.5^{\circ} / \mathrm{sec}$ to $170^{\circ} / \mathrm{sec}$. It holds for oscillation angles up to $100^{\circ}-140^{\circ}$. With larger rotational angles, saturation is reached. With small stimulus angles, a surprisingly high threshold $\left(5^{\circ}-8^{\circ}\right)$ was observed in our experiments. This may reflect the unphysiological combination of visual roll stimuli without corroborating vestibular and proprioceptive inputs normally present when body sway produces visual stimulation. Under natural conditions, the visual feedback about spontaneous sway stabilizes body posture by integrating rotational velocity over stimulus duration which is equal to rotational angle.
\end{abstract}

Roll motion of a large visual scene has been shown to induce tilt of the perceived vertical and postural upright toward the same direction (Dichgans, Held, Young, \& Brandt, 1972). It was assumed that both phenomena reflect the dependency of the central estimate of orientation with respect to gravity on seen motion mainly from the retinal periphery (Held, Dichgans, \& Bauer, 1975). This assumption is supported by the well-known stabilization of spontaneous involuntary postural sway through vision (Romberg, 1846). That motion, and not only the successive perception of contour orientation, is important is suggested by the observation of Amblard and Cremieux (1976), who found less stabilization if the visual environment was briefly flashed at rates below $6 / \mathrm{sec}$.

The following consideration illustrates the relation between the illusions mentioned above and their behavioral equivalent. Postural sway, for example to the right, causes relative motion of the visual scene to the left. If this relative motion is simulated under laboratory conditions, tilt of the subjective vertical to the left is elicited and causes a corrective postural response from right to left. This, in the laboratory, where the initial real body tilt is missing, causes falling (Dichgans, Brandt, \& Held, 1975; Dichgans, Mauritz, Allum, \& Brandt, 1976), whereas under real-life conditions, a righting response occurs.

The question then arises as to what the key parameters involved in this feedback stabilization

This research was supported by the "Deutsche Forschungsgemeinschaft" (Sonderforschungsbereich 70, "Hirnforschung und Sinnesphysiologie'). process are. The angle of visually induced tilt of subjective vertical has been demonstrated to depend on the speed and duration of the rolling stimulus (Held et al., 1975). Regarding this, we examined whether those parameters were of invariant significance, or whether it is, rather, the product of speed and duration which amounts to the angle (amplitude) of seen motion that is processed for perceived vertical. Since postural sway and the consequent visual stimulus may be broken down into sinusoids of different amplitudes and frequencies, we used sinusoidal roll motions of a hemispherical screen to test this alternative, and applied stimuli of constant frequency, constant peak velocity, or constant amplitude (in visual angle), each time varying the other two parameters correspondingly.

\section{METHODS}

\section{Subjects}

A total of 29 healthy subjects between 18 and 39 years of age took part in the experiments. Refractive errors were corrected in 7 of them.

\section{Apparatus}

Tilt of the subjective vertical was induced by a visual display which rotated in front of the observer. The display consisted of a half-spherical dome (radius $40 \mathrm{~cm}$ ) mounted so that its axis could be aligned with the subject's line of sight. The inner surface of the dome was covered by randomly distributed colored dots of different sizes on a white background. The dome could be illuminated diffusely from behind. It covered the entire visual field of the observer up to $80^{\circ}$ peripherally, and could be rotated about its axis at servo-controlled angular velocities ranging from $.5^{\circ} / \mathrm{sec}$ to $170^{\circ} / \mathrm{sec}$.

The subjects stood on a stable force-measuring platform (Kistler) that recorded the motion of their centers of force. A small disk, 
whose position could be controlled by the subject manipulating a potentiometer, was mounted coaxially with the dome just in front of its inner surface. The disk was bisected by a narrow line with a fixation point in the center. The subjects were instructed to maintain this diameter oriented to their subjective vertical.

The stimulus velocity profile and the potentiometer output were recorded on paper and on FM tape. Lateral sway was also recorded, but the results will be reported separately.

\section{Stimuli}

Stimuli employed were sinusoidal rotations of the dome at discrete frequencies in the range from $.1 \mathrm{~Hz}$ to $1 \mathrm{~Hz}$. Peak velocities ranged from $5^{\circ} / \mathrm{sec}$ to $170^{\circ} / \mathrm{sec}$ and oscillation angles from $7.5^{\circ}$ to about $360^{\circ}$. Among the three stimulus parameters, one was always maintained constant, while the other two varied according to the following simple equation:

$$
\Theta=\frac{V_{p}}{2 \pi v}
$$

where $\theta$ is the oscillation angle in degrees, $V_{p}$ is the peak velocity in degrees/second, and $v$ the frequency of rotation in hertz. The parameters of the individual experiments may be taken from the abscissae of Figures 1, 4, and 5.

Usually, the subjective responses to eight cycles were averaged using a Nicolet 1072 computer or using a graphic method of superposition and of calculating the average response amplitudes. The first two or three cycles were not used since they are more variable than the following ones. The amplitude of the fundamental wave of the averaged individual SV displacement as well as the phase angle in relation to the stimulus were thus determined in relation to the initial calibration.

\section{RESULTS}

\section{Sinusoidal Rotations at Constant Frequencies}

In an initial series of experiments, the influence of increasing peak velocities $\left(10^{\circ}-80^{\circ} / \mathrm{sec}\right)$ upon SV-tilt was examined at varying frequencies $(.1$ to $.3 \mathrm{~Hz}$ ) in five subjects. These frequencies were chosen after preliminary experiments had shown that within this frequency range subjects are able to consistently adjust the test edge with the least amount of distortion.

The average results and standard deviations of these measurements are plotted in Figure 1. The increase of induced tilt with increasing peak velocities for sinusoidal stimuli is similar to that found with constant velocity rotations (Dichgans et al., 1972). The magnitude of SV tilt, however, seems to also depend on frequency, and the peak velocity at which tilt is saturated seems to increase with increasing oscillation frequencies. It occurs at $30^{\circ} / \mathrm{sec}$ for constant-velocity stimuli (Dichgans et al., 1972), at $60 \% \mathrm{sec}$ for $.1-\mathrm{Hz}$ oscillations and beyond the velocity range studied for $.3-\mathrm{Hz}$ oscillations (see Figure 1). These differences were then thought to be due to the fact that, with sinusoidal motion, the peak velocity is reached for only short time intervals.

A comparison of the tilt induced by identical angles of rotation but accomplished by different combinations of stimulus frequencies and peak velocities (see upper scale in Figure 1) suggests that

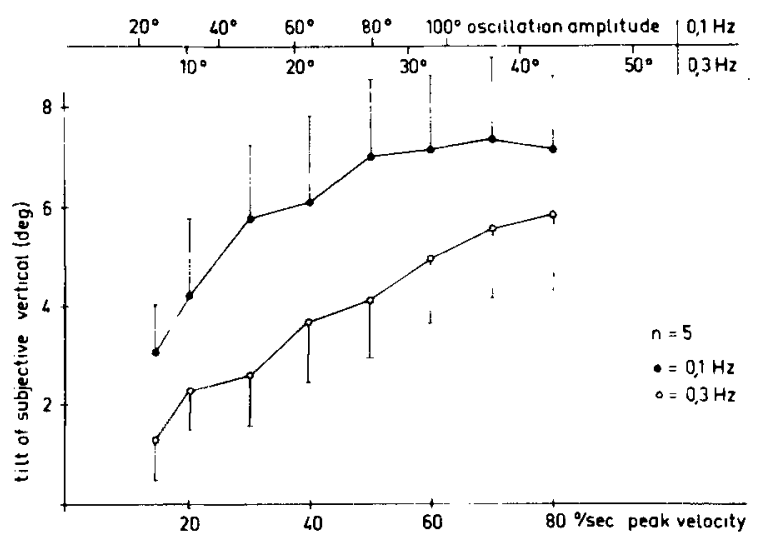

Figure 1. SV tilt in relation to increasing peak velocities at $.1 \mathrm{~Hz}$ (black dots) and $.3 \mathrm{~Hz}$ (open circles). Average of five subjects with corresponding standard deviations. The additional scale at the top indicates the corresponding oscillation amplitudes.

the angle of SV tilt is, indeed, a function of stimulus oscillation angles.

To further examine the hypothesis of an angle dependence, different rotational angles of $15^{\circ}, 30^{\circ}$, $45^{\circ}, 60^{\circ}$, and $90^{\circ}$ were presented to another five subjects at the same two preset frequencies $(.1$ and $.3 \mathrm{~Hz}$ ) by changing the peak velocity between $9.4^{\circ} / \mathrm{sec}$ and $170^{\circ} / \mathrm{sec}$ according to Equation 1. Figure 2a shows original recordings from one subject. The averaged results from all five subjects plotted in Figure $2 b$ show a linear relationship between SV displacement and the logarithm of the stimulus oscillation amplitudes. No significant difference is observed between the curves at two different frequencies (Figure 2b). The logarithmic relationship of the averages can be described by $\mathrm{SV}=-6.29+3.50$ ln $\Theta, \mathrm{r}=.92$ for $.1 \mathrm{~Hz}$; and by $\mathrm{SV}=-8.34+$ $4.10 \ln \Theta, r=.95$ for $.3 \mathrm{~Hz}$ stimuli. As may be seen from Figure 2, the regression line meets the abscissa at about $7^{\circ}$, which, provided that this relationship holds at the lower limit, indicates the threshold. A similar stimulus displacement threshold (average $5.3, \mathrm{SD}=.82$ ) is suggested by the data plotted in Figure 3.

\section{Sinusoidal Rotations at Constant Peak Velocities}

To further investigate whether SV displacement is solely determined by the angle of stimulus oscillation, three additional sets of experiments have been performed in which peak velocity was kept constant at $15 \% / \mathrm{sec}, 25^{\circ} / \mathrm{sec}$, or $50 \% / \mathrm{sec}$, and frequencies were varied from .01 to $.3 \mathrm{~Hz}$ in order to obtain the desired oscillation angles. The results obtained with $15^{\circ} / \mathrm{sec}$ peak velocity in five subjects are illustrated in Figure 3. The average angle of visually induced SV tilt plotted in relation to the stimulus oscillation angle is shown to again increase linearly with the logarithm of the rotational angle in all subjects up to 
A

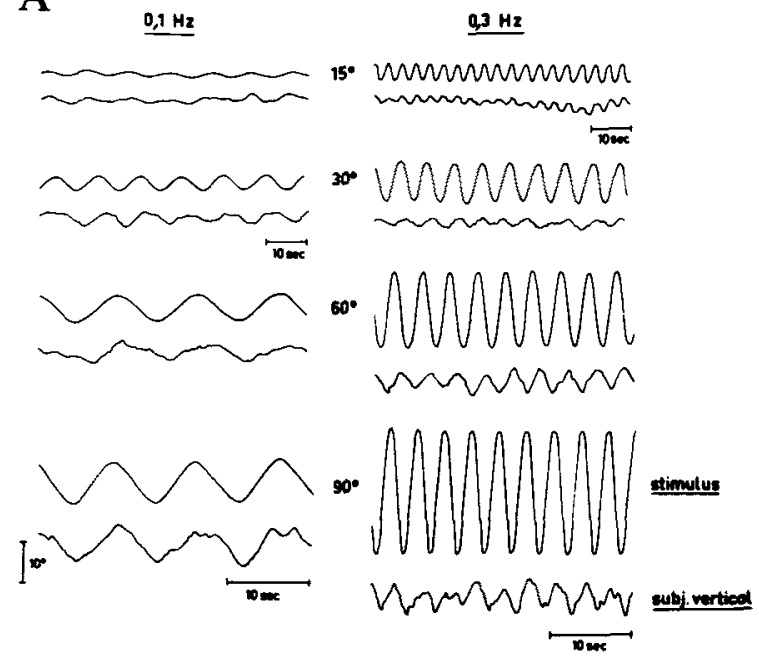

B

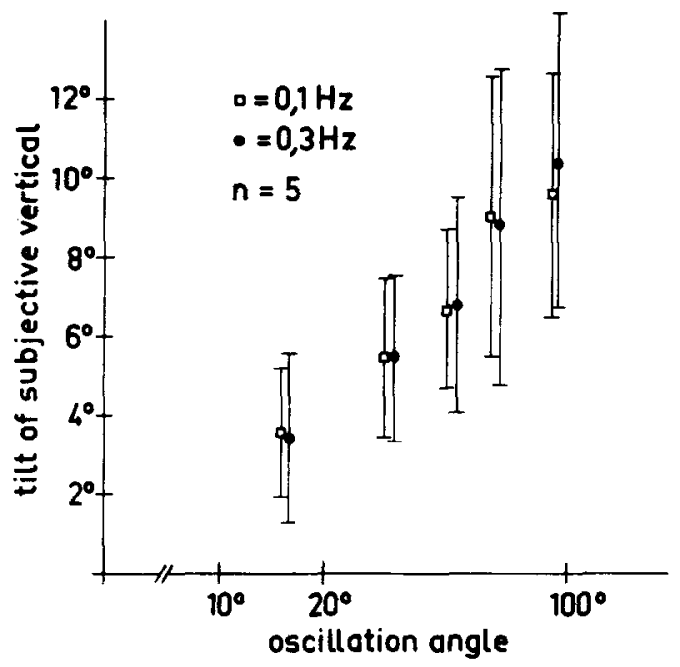

Figure 2. (a) Original recording of $\mathrm{SV}$ tilt in one subject at oscillation angles of $15^{\circ}, 30^{\circ}, 60^{\circ}$, and $90^{\circ}$ and stimulus frequencies of $.1 \mathrm{~Hz}$ (left) and $.3 \mathrm{~Hz}$ (right). Note the different time scales. (b) $\mathrm{SV}$ tilt in relation to increasing oscillation angles $\left(15^{\circ}\right.$, $30^{\circ}, 45^{\circ}, 60^{\circ}$, and $90^{\circ}$, abscissa) at .1 and $.3 \mathrm{~Hz}$. Average and SD of five subjects.

the largest stimulus angles (approximately $80^{\circ}$ ), produced by the lowest frequency used in this experiment $(.028 \mathrm{~Hz})$. The intraindividual deviation from the linear function is small. This is indicated by the correlation coefficient (r) given for each subject. The interindividual variability of the slopes, which vary from 2 to 9 , is considerable. This indicates the variability in gain of the visual influence upon orientation vs. gravity among subjects. Taken together, these results allow the conclusion that the SV tilt is proportional to the logarithm of the roll vection oscillation angle and depends only indirectly on stimulus velocity and stimulus duration.

\section{The Range of the Logarithmic Relation}

The range of oscillation angles over which this logarithmic relationship holds can be estimated from the results presented in Figure 4. Here, peak velocity was maintained at $50^{\circ} / \mathrm{sec}$ (black dots) and frequencies were varied from .02 to $.3 \mathrm{~Hz}$. The figure shows a saturation of the angle dependence between $100^{\circ}$ and $140^{\circ}$ of oscillation angle, which corresponds to $.08-.1 \mathrm{~Hz}$ at this peak velocity. Experiments with $25^{\circ} / \mathrm{sec}$ (triangles) peak velocity show a similar saturation limit and thereby confirm the validity of the exclusive oscillation angle dependence. The marked increase of the response variability at higher oscillation angles comprises a large interindividual variability of the saturation angle (gain) and an increased intraindividual uncertainty at these larger oscillation angles.

\section{Frequency Characteristic of SV Tilt}

The preceding data demonstrated that the SV-tilt is invariably related to the oscillation angle of the sinusoidal roll-motion stimulus. In terms of an analytical approach, an integrative system is suggested. This suggestion is supported by the phase data given in addition to the amplitude data for constant peak velocity stimuli $\left(15^{\circ} / \mathrm{sec}\right)$ of various frequencies $(.01 \mathrm{~Hz}$ up to $.5 \mathrm{~Hz})$, which are plotted in a conventional BODE diagram in Figure 5. As would be expected from an integrative system, the amplitude progressively diminishes at higher frequencies (with frequencies exceeding $.1 \mathrm{~Hz}$, this diminution amounts to $60 \mathrm{~dB}$ per decade). The phase lag slowly increases from $25^{\circ}$ at $.01 \mathrm{~Hz}$ to about $90^{\circ}$ at $.5 \mathrm{~Hz}$. Thus, the lower the frequency, the less perfect the integration.

\section{Periodic Resetting at Constant Velocity and Low-Frequency Stimulation}

In addition to the saturation effect observed at

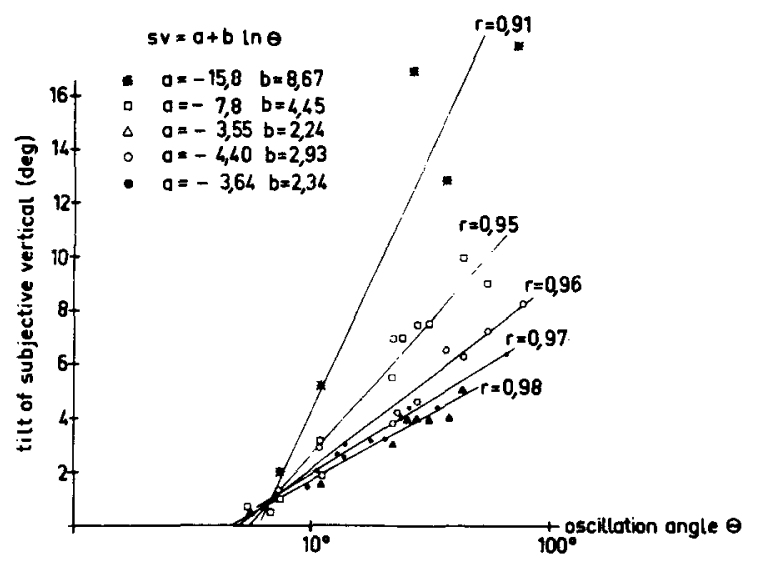

Figure 3. SV tilt dependent on oscillation angle (abscissa) with sinusoidal stimuli of constant peak velocity $\left(15^{\circ} / \mathrm{sec}\right)$ and varying frequency. Individual data from five subjects illustrating the high variability in slope. 


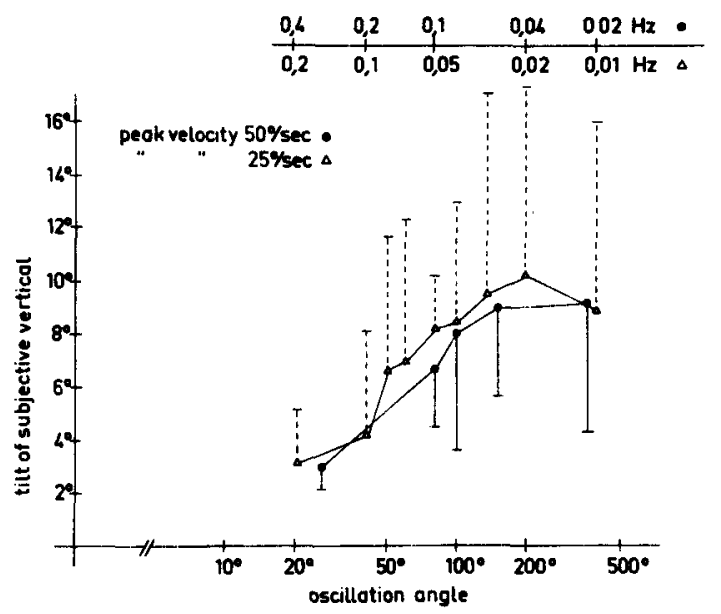

Figure 4. SV tilt dependent on oscillation angle (abscissa) with sinusoidal stimuli of constant peak velocity (triangles, $25 \%$ sec; black dots, $50^{\circ} / \mathrm{sec}$ ) and varying frequency. Average and $\mathrm{SD}$ of five subjects. Note the saturation at higher rotational angles.

oscillation angles exceeding $100^{\circ}-140^{\circ} / \mathrm{sec}$ (Figure 4), there is another limitation to the rule of angle dependence. At very low frequencies, a periodic distortion appears which is more clearly observed with continuous stimulus motion (Figure 6a). This distortion consists of periodical breakdowns of both the roll vection sensation and the SV tilt. Figure $6 a$ shows a concomitant resetting of the deviation of posture invariably observed with roll vection stimuli (Dichgans et al., 1976). A systematic investigation of the periodicity and amplitude was performed with 15 subjects using constant velocity stimuli of $50^{\circ} / \mathrm{sec}$. Optokinetically induced tilt of SV increases to its maximum within an average latency of $11.6 \mathrm{sec}$ (SD 3.74) and is then maintained over several seconds. The observed breakdown always occurs abruptly. The cycle time between two consecutive resets was found to remain rather constant over a 10-min - period of stimulation in 3 subjects (Figures $6 \mathrm{~b}$ and $6 \mathrm{c}$ ). A systematic trend was not observed over this period. Subsequently, 15 -subjects were tested for the duration of their first resetting period. This was found to last an average of $30.2 \mathrm{sec}$ (SD 4.76).

Only slight adaptation in displacement amplitude was found during the initial cycles. Later, the SV displacement remained constant. During the resetting periods, SV did not completely return to objective upright, but showed a residual displacement toward stimulus motion. This residual displacement continued to increase beyond the time of adaptation.

\section{DISCUSSION}

Our results confirm the hypothesis that for sinusoidal rotations around the roll axis, the tilt of the $\mathrm{SV}$ is related to the oscillation angle of the visual stimulus, i.e., to the product of peak velocity and oscillation period. These results are in agreement with the data of Dichgans et al. (1972), who demonstrated a velocity dependence, and with the report of Held et al. (1975), who found a time dependence on the effect induced by a constant-velocity stimulus. Constant-velocity stimuli, however, disclose some limitations on this dependence on stimulus angle. The maximal time over which stimulus velocity may be integrated has been found to average $11.6 \mathrm{sec}$ in 15 subjects, which is considerably shorter than the interval estimated by Held et al. on the basis of recordings from a few subjects while using

\section{SV-settings}

\section{(peak velocity of stimulus $15 \% \mathrm{sec}$ )}
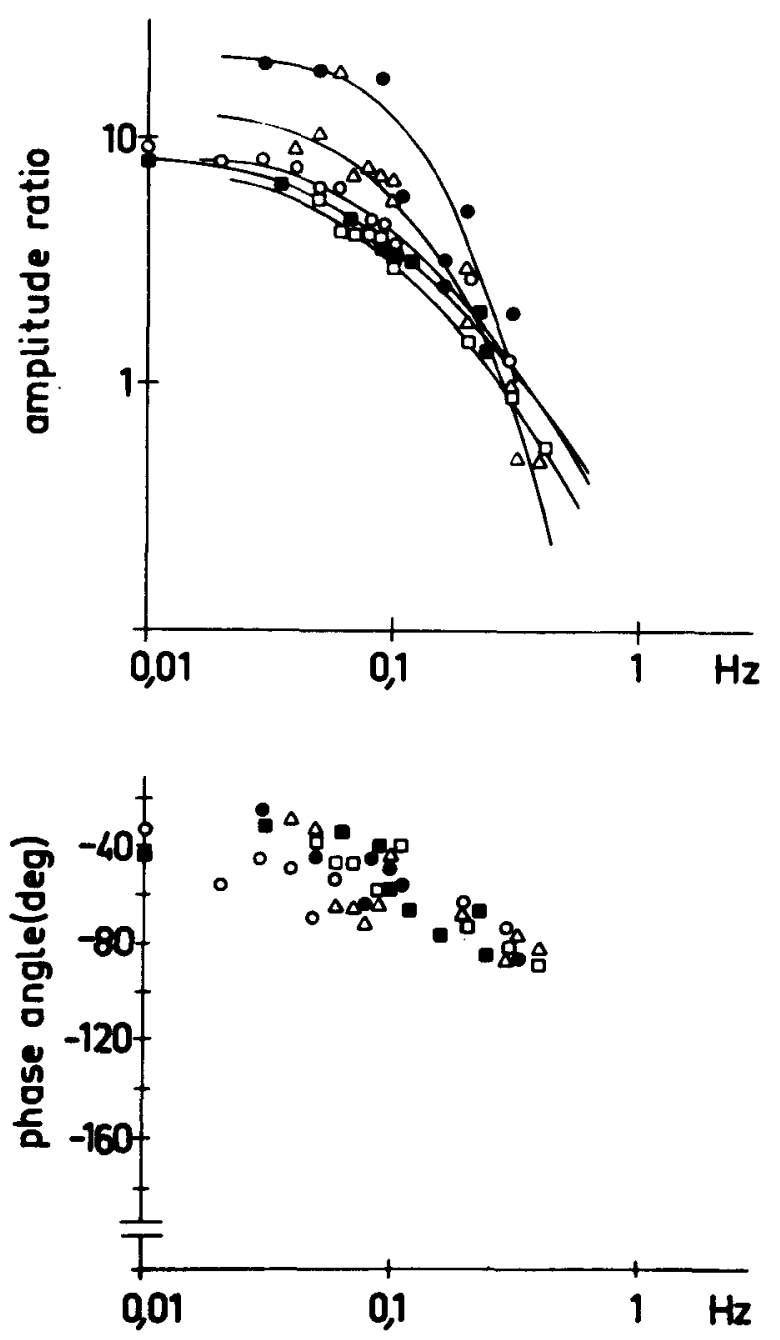

Figure 5. Amplitude ratio (angle of SV displacement vs. peak stimulus velocity, $15 \%$ sec) in relation to stimulus frequency (BODE diagram). The lower half of the figure displays the phase relationship as dependent on stimulus frequency. 


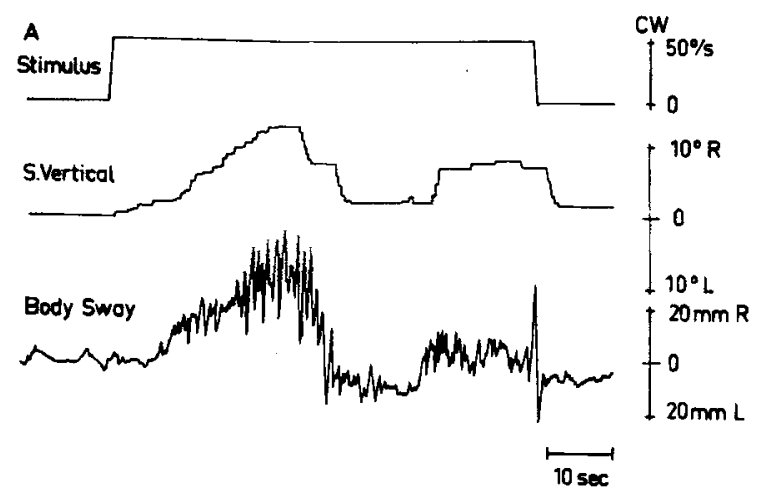

B SV-resetting

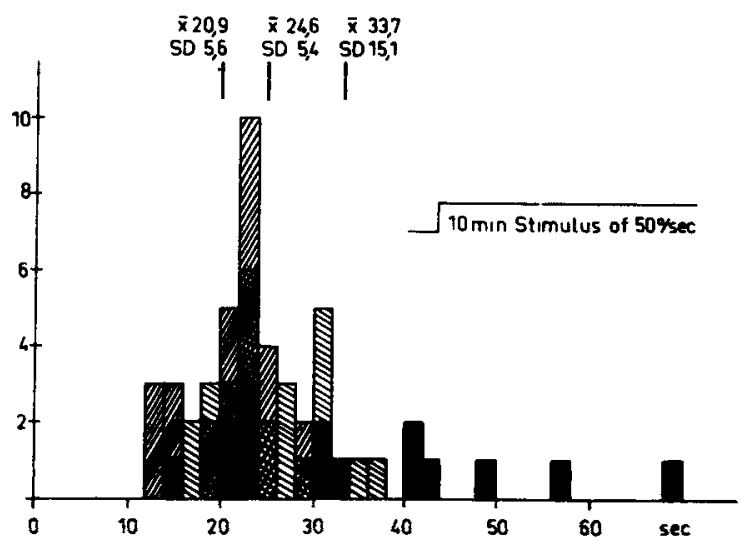

C

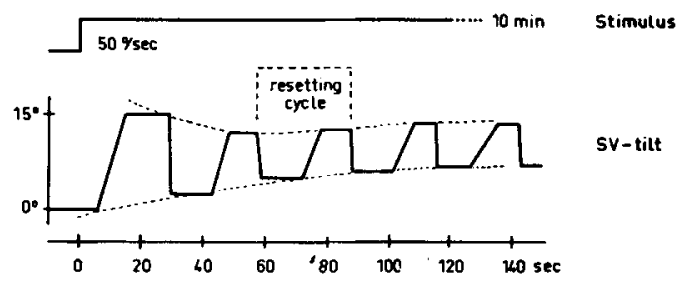

Figure 6. (a) $\mathrm{SV}$ tilt in response to constant-velocity rotation $\left(50^{\circ} / \mathrm{sec}\right)$. Original recording in one subject. Note the breakdown of $\mathrm{SV}$ displacement and the concomitant deviation of posture. The discontinuous stepwise tracking of SV reflects the subject's tracking strategy. The perceived SV displacement, however, changes continuously. The tracing of posture displays the initial spontaneous sway and an increase in roughly .5-Hz lateral oscillations with increasing deviation from real upright during the stimulation period (Dichgans et al., 1976). (b) Histogram of consecutive resetting intervals in three subjects during a 10 -min stimulation period with $50^{\circ} / \mathrm{sec}$ constant velocity. The frequency of each interval (ordinate) is plotted vs. the duration of a breakdown cycle (abscissa). The average duration is indicated for each subject. (c) Schematic drawing of the fluctuating displacement of $\mathrm{SV}$ during long-lasting constant-velocity stimulation. Note the early adaptation in induced displacement amplitude and the slow increase in residual displacement during the resting periods. monocular stimulation and a limited peripheral visual field. The saturation velocity $\left(30^{\circ} / \mathrm{sec}\right)$ observed by these authors allows the estimation of the roll angle at which induced SV displacement becomes saturated with a constant-velocity stimulus. The resulting value of $300^{\circ}$ contrasts with the results of our experiments with sinusoidal stimuli in which the maximum tilt effect was reached at smaller oscillation angles of $100^{\circ}$ to $140^{\circ}$. This may again be due to the different experimental conditions. Our results do not indicate that the saturation angle increases with decreasing oscillation frequency (Figure 4).

The large interindividual variability in induced SV tilt (Dichgans et al., 1972; Held et al., 1975) and in the postural response to moving visual stimuli (de Wit, 1972; Dichgans et al., 1976; Lestienne, Berthoz, Mascot, \& Koitcheva, 1976) is reflected in interindividual variations in the slope (Figure 3) and the large standard deviations in Figure 4. The slope of the logarithmic relations varies between 2 and 9 . It may be assumed that different subjects rely to varying degrees on visual information for the perception of spatial coordinates and for postural stabilization.

The data reported here largely confirm the hypothesis about the possible basis of visual stabilization of posture. There is, however, an unexpectedly high threshold for this supposed functional significance (Figures 2 and 3). Physiological body sway amplitudes are below $2^{\circ}$ to either side of vertical (Dichgans et al., 1976; Nashner, 1970; Thomas \& Whitney, 1959). Visually induced SV displacement, however, in our experiments required a threshold stimulus displacement of $2.5^{\circ}$ to $4^{\circ}$ to either side. Below that threshold, no consistent tilt of subjective vertical was observed. This high threshold may probably be explained by an experimental artifact, since here, in contrast to natural conditions, visual afferences on the one hand and vestibular and proprioceptive afferences on the other are contradictory rather than confirming. In the experiment, small visual effects are therefore presumably suppressed by contradictory vestibular and proprioceptive inputs.

The strong asymmetry of the visually induced SV tilt dependent on whether or not steady head tilt and seen motion are in functionally corresponding (opposite) directions or not (Dichgans et al., 1975; Dichgans, Diener, \& Brandt, 1974) supports this assumption. Static head tilt, however, provides only steady state stimulation to the otolith, whereas actual head motion adds dynamic inputs from both the graviceptors and the semicircular canals. The fact that the visually induced sensation of ego-motion is facilitated by corresponding vestibular stimuli was discussed earlier by Brandt, Dichgans, and Büchele (1974) with reference to their circular vection experiments. These authors emphasized the necessity of this cooperative facilitation if one wants to attribute any behavioral significance to these results. 
The oscillation angle dependence of SV displacement represents an interesting fact in its own right. Its functional significance for the stabilization of posture is indicated by the invariant observation of a head and body displacement toward the same side whenever the subjective vertical is displaced by optokinetic stimuli. Head displacement almost always clearly exceeds the lateral displacement of the vertical body axis.

The visual feedback signal during physiologic body sway contains both linear and rotational components with a preponderance of translational signals, whereas under our experimental conditions, with the axis at the level of the head, rotational components are exaggerated. This may explain the larger tilt of the head compared to the tilt of the body upright. In contrast to the anterior-posterior direction, lateral body sway must be described by a multilink model and not just as an inverted pendulum. The multilink model would predict that our results for subjective vertical displacement are most valid for stabilization of the head segment, whereas segmental motion about the lower axis, e.g., the ankles, elicits a visual motion feedback with progressively increasing linear motion components that have been studied by Lestienne, Soechting, and Berthoz (1977).

Interpretations of the total postural body reaction to the sinusoidal visual roll motion stimuli are in progress. So far, it seems that the simple semilogarithmic relationship does not hold for the overall response of the multilink system. This, as it is assumed for thresholds of SV displacement, may again be due to conflicting afferent information with different weighting of the separate control signals for-the organization of postural stabilization of each segment.

\section{REFERENCES}

Amblard, B., \& Cremieux, J. Rôle de l'information visuelle du mouvement dans le maintien de l'équilibre. Aggressologie, 1976, 17c, $25-37$.

Brandt, Th., Dichgans, J., \& Büchele, W. Motion habituation: Inverted self-motion perception and optokinetic afternystagmus. Experimental Brain Research, 1974, 21, 337-352.

DE WIT, G. Optic versus vestibular and proprioceptive impulses measured by posturometry. Aggressologie, 1972, 13b, 75-79.

Dichgans, J., Brandt, Th., \& Held, R. The role of vision in gravitational orientation. Fortschritte der Zoologie, 1975, 23, 255-263.

Dichgans, J., Diener, H.-C., \& Brandt, Th. Optokinetic graviceptive interaction in different head positions. Acta Otolaryngologica, 1974, 78, 391-398.

Dichgans, J., Held, R., Young, L., \& Brandt, Th. Moving visual scenes influence the apparent direction of gravity. Science, $1972,178,1217-1219$.

Dichgans, J., Mauritz, K.-H., Allum, J. H. J., \& Brandt, Te. Postural sway in normals and atactic patients: Analysis of the stabilizing and destabilizing effects of vision. Aggressologie, 1976, 17c, 15-24.

Held, R., Dichgans, J., \& Bauer, J. Characteristics of moving visual scenes influencing spatial orientation. Vision Research, $1975,15,357-365$.

Lestienne, F., Berthoz, A., Mascot, J.-C., \& Kortcheva, V. Effets posturaux induits par une scène visuelle en mouvement linéaire. Aggressologie, 1976, 17c, 37-46.

Lestienne, F., Soechting, J., \& Berthoz, A. Postural readjustments induced by linear motion of visual scenes. Experimental Brain Research, 1977, 28, 363-384.

NASHNER, L. M. Sensory feedback in human posture control. Scientific Doctoral Thesis, MTV 70-3, Cambridge, Mass: Man Vehicle Lab., MIT, 1970.

RomBerg, M. H.Lehrbuch der Nervenkrankheiten des Menschen. Berlin: A. Duncker, 1846.

Thomas, D. P., \& Whitney, R. J. Postural movements during normal standing in man. Journal of Anatomy, 1959, 93, 524-529.

(Received for publication April 18, 1977; revision received August 15, 1977.) 